\title{
Use of Fast-Responding Voltage-Sensitive Dyes for Large-Scale Recording of Neuronal Spiking Activity with Single-Cell Resolution
}

\author{
William N. Frost, Jean Wang, Christopher J. Brandon, \\ Caroline Moore-Kochlacs, Terrence J. Sejnowski, and Evan S. Hill
}

\subsection{INTRODUCTION}

Efforts to understand how the brain works require tools for observing circuits in action. Conventional electrophysiological methods can monitor no more than a few neurons at a time, providing a severely restricted perspective on network function. Optical recording with fast voltage-sensitive dyes (fVSDs) offers a way to overcome this limitation, by revealing the action potentials of dozens to hundreds of individual neurons during behaviorally relevant motor programs (Fig. 5.1). The availability of methods for monitoring large-scale brain activity with single-cell and sub-millisecond resolution is likely to advance our understanding of network function far beyond that attained using traditional methods (Yuste 2008).

It has been three decades since fVSDs were first used to monitor the simultaneous firing of multiple individual neurons (Grinvald et al. 1977; Salzberg et al. 1977). Although most such studies have used invertebrate preparations, particularly those with large neurons whose action potentials fully invade the soma (Boyle et al. 1983; London et al. 1987; Cohen et al. 1989; Zecevic et al. 1989; Nakashima et al. 1992; Nikitin and Balaban 2000; Zochowski et al. 2000a; Brown et al. 2001; Kojima et al. 2001; Frost et al. 2007; Wu et al. 1994a), a few studies have accomplished this in vertebrate preparations, such as the enteric nervous system ganglia (Neunlist et al. 1999; Obaid et al. 1999; Vanden et al. 2001; Schemann et al. 2002).

Given the enormous potential of optical recording with fVSDs for studying neural networks, why have not more laboratories adopted this approach? A key reason is that the optical signals corresponding to action potentials in individual neurons are miniscule - often ranging from 0.001 to less than 0.0001 of the resting light level - making their detection quite challenging. Another reason is the difficulty of combining high resolution imaging with intracellular recording from multiple neurons. Although such combined methodology would be highly useful for circuit mapping, the light-efficient compound microscopes used for imaging lack stereopsis, making integrating multiple intracellular electrodes extremely difficult. Ideally, one could penetrate and drive a known neuron, image its followers, and then penetrate those followers with a second electrode to test for a direct synaptic connection. A third difficulty with using optical recording for network studies is the large, unwieldy data sets generated. Individual detectors of either camera or photodiode array systems often record multiple neurons, yielding mixed signals. Conversely, multiple detectors often record the same neuron, yielding redundant traces. As a result, it can be very difficult to know the number of neurons included in the optical data set.

Fortunately, it is possible to overcome all these difficulties. Here we describe procedures for obtaining satisfactory signal-to-noise ratio using fVSDs, for easier integration of sharp electrodes into imaging experiments, and for the transformation of raw data sets of mixed and redundant traces into new sets containing a single neuron per trace. This discussion is particularly relevant for investigators considering large-scale optical recording with fVSDs with single cell resolution, such as is readily achievable in invertebrate ganglia and in certain vertebrate peripheral nervous system preparations. Here we focus primarily on the use of photodiode arrays.

\subsection{CHOICE OF FLUORESCENCE VS. ABSORBANCE FAST VOLTAGE SENSITIVE DYES}

Fast voltage-sensitive dyes come in two main types: absorbance and fluorescence (Ebner and Chen 1995; Zochowski et al. 2000b). Both change their light response linearly with membrane potential, and do so fast enough to trace out each action potential. Generally speaking, absorbance dyes have been preferred for network studies, in part due to their lower toxicity at the high light levels needed to attain sufficient signal-to-noise ratio. Direct comparisons in the vertebrate brain slice have found absorbance dyes to yield larger signal-tonoise with less phototoxicity than the tested fluorescence dyes (Jin et al. 2002; Chang and Jackson 2003). Two such absorbance dyes, RH155 and RH482, have been applied to preparations ranging from invertebrate ganglia (Yagodin et al. 1999), to vertebrate slice (Senseman 1996; Momose-Sato et al. 1999; Yang et al. 2000), to cell culture preparations (Parsons et al. 1991). We have similarly used both dyes successfully in Tritonia and Aplysia. Recently, fast fluorescent dyes have been developed and used that have lower phototoxicity and other desired qualities than previous dyes (for example, see Obaid et al. 2004; Carlson and Coulter 2008). We have not yet tried these on our invertebrate preparations.

Recently developed calcium dyes also allow imaging of the firing of hundreds of individual neurons simultaneously in the

William N. Frost, Jean Wang, Christopher J. Brandon and Evan S. Hill • Department of Cell Biology and Anatomy, The Chicago Medical School, Rosalind Franklin University of Medicine and Science, North Chicago, IL 60064, USA

Caroline Moore-Kochlacs and Terrence J. Sejnowski - Howard Hughes Medical Institute, The Salk Institute for Biological Studies, La Jolla, CA 92037, USA Terrence J. Sejnowski • Division of Biological Sciences, University of California San Diego, La Jolla, CA 92093, USA 


\section{A Conventional intracellular recording}

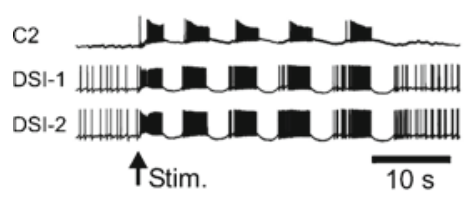

\section{B Optical recording}

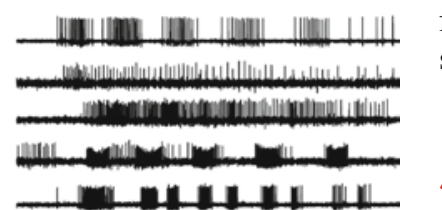
randu|di||

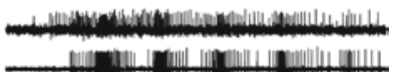

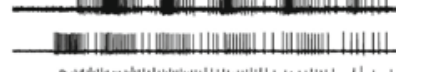



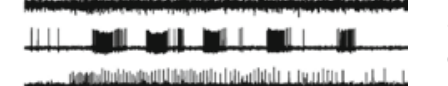
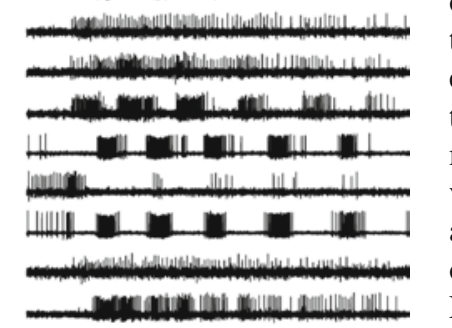

ЩبШ
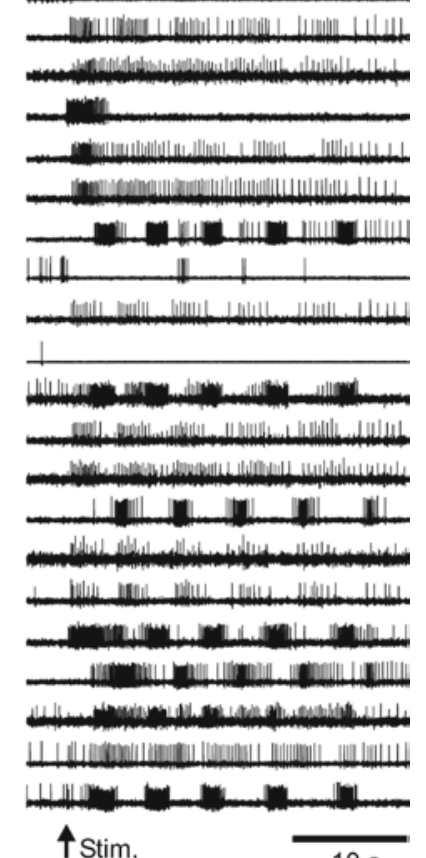

$10 \mathrm{~s}$

FIGURE 5.1. Imaging with fast voltage sensitive dyes allows simultaneous recording of the individual action potentials generated by large numbers of neurons. (A) Conventional sharp electrode intracellular recording of the Tritonia swim motor program. A $1 \mathrm{~s} 10 \mathrm{~Hz}$ stimulus to Pedal Nerve 3 triggered a 5 cycle swim motor program, recorded in the C2 and DSI interneurons of the swim central pattern generator. Experiments using sharp electrodes are typically limited to recording 3-4 neurons simultaneously. (B) Optical recording of the Tritonia swim motor program, after processing with Independent Component Analysis, showing 41 of the 63 neurons detected. A $2 \mathrm{~s} 10 \mathrm{~Hz}$ stimulus to Pedal Nerve 3 was used to trigger this 5 cycle motor program, which was recorded with a RedShirtImaging NeuroPDA-III 464 photodiode array and the fast absorbance voltage sensitive dye RH155.

vertebrate brain in vivo (Stosiek et al. 2003; Kerr et al. 2005; Sato et al. 2007; Takahashi et al. 2007; Greenberg et al. 2008). Although these dyes can resolve isolated single action potentials,

the slow dynamics of calcium resequestration means that action potentials occurring in trains and bursts merge into a single waveform. Fast VSDs thus remain superior for network studies requiring single action potential resolution in multiple neurons.

\subsection{CHOICE OF IMAGING SYSTEM}

Most imaging systems employ either a camera or photodiode array. Both designs operate as a spatial array of independent sensors that convert light to voltage, with the entire array sampled at a fixed frequency. Two systems we have tried by RedShirtImaging are the NeuroCMOS-SM128 camera and the NeuroPDA-III photodiode array. The CMOS-SM128 has 16,384 independent sensors arranged on a $128 \times 128$ chip, with the full set sampled at $2,500 \mathrm{~Hz}$. Using the RedShirtImaging Neuroplex software, the data can be viewed either as a camera image of the preparation at different points in time, with pixel color or grayscale brightness indicating the membrane potential of that location, or as a set of up to 16,384 voltage traces (one trace per pixel). The latter can be spatially averaged using either grid-based binning or user-outlined regions of interest, such as single neurons. By contrast, the RedShirtImaging NeuroPDA-III is organized as a hexagonal array of 464 sensors (Fig. 5.2), with the full set sampled at 1,600 Hz. The PDA data are shown as miniature traces organized on screen in the positions of the diodes that recorded them. Because the PDA does not have the spatial resolution to form a useful image, the 464 miniature traces are superimposed on an image of the preparation taken by a separate digital camera that can also be used to focus the PDA on the neurons of interest. As with the camera system, diodes can be selected individually or in groups for display of their recorded voltage traces, raw or filtered, individual or spatially averaged, in a separate window in Neuroplex (Fig. 5.3).

Cameras and photodiode arrays have different strengths and weaknesses. Cameras such as the CMOS-SM128 have sufficient pixel density to provide a recognizable image, which simplifies focusing the imager and determining which regions and/or neurons gave rise to the recorded signals. On the negative side, the camera's larger sensor number leads to filesizes approximately 50x larger than those acquired by the PDA for the same recording duration. Another difference regards the sensitivity of the two types of systems. While both systems digitize the data with 14 bit resolution (the CMOS system in the camera, the PDA via an A/D board in the computer), the NeuroPDA-III gains nearly seven additional bits of effective resolution by first AC-coupling each trace to subtract the resting light level, and then amplifying it by $100 \times$ before digitization. This makes PDAs more suitable for detecting the smaller signals provided by absorbance dyes if digitization resolution is a limiting factor, a point made by several others (Sinha and Saggau 1999; Vanden Berghe et al. 2001; Kosmidis et al. 2005). It is worth noting, however, that AC-coupled PDAs are unsuited for studies of slow events, such as slow EPSPs. The majority of our remaining discussion concerns our experience with the NeuroPDA-III.

\subsection{TWO MICROSCOPE DESIGNS FOR OPTICAL RECORDING}

We use two different microscope designs, depending on the degree to which intracellular electrodes will be used in the experiment. In both systems (Fig. 5.4), the microscope is mounted on an EXFOBurleigh motorized translator attached to a Burleigh Gibraltar stage 
FIGURE 5.2. How the RedshirtImaging NeuroPDA-III sees and displays the data. (A) Hexagonal face formed by the ends of the bundle of 464 fiber optics upon which the image of the preparation is focused. The other ends of these fiber optics are individually glued to their corresponding diodes of the 464 photodiode array. (B) Depiction of an RH155-stained Tritonia pedal ganglion focused onto the array input port. Individual neurons of many different sizes are evident. The largest neurons will be recorded by up to several dozen photodiodes, leading to redundancy in the recordings, while the smallest will be detected by just one or two photodiodes. (C) Data display in Neuroplex. As soon as the data are acquired, Neuroplex displays them as miniature traces organized according to the position of each acquiring diode in the array. Shown is a $35 \mathrm{~s}$ optical recording of a swim motor program, obtained from a different preparation than the one photographed in panel (B). Nearly 50 diodes detected a single large neuron located in the upper right portion of the array. Clicking on specific traces expands them in a separate window in Neuroplex (see Fig. 5.3).
FIGURE 5.3. Superimposing an image of the preparation with the diode array makes it easy to inspect the firing of different neurons. The left panel shows an image of the dorsal surface of the pedal ganglion, superimposed in Neuroplex with a display of the 464 acquired optical traces in their corresponding diode positions (green lines). The right panel shows how clicking on diodes of interest displays their optical data. These are filtered, unaveraged recordings from single diodes, each showing the firing recorded from the indicated ganglion location. A five cycle swim motor program was elicited by a $10 \mathrm{~Hz}, 1 \mathrm{~s}, 10 \mathrm{~V}$ stimulus to Pedal Nerve 3, delivered at the arrow.


that provides a large stable platform for mounting the manipulators, recording chamber, suction electrodes, bath ground, bath temperature probe, optional epi-illumination, perfusion tubes, and cooling system used in our Tritonia experiments. The translator allows penetration of a neuron in one ganglion, and then subsequent movement of the microscope and PDA without disturbing the impalement, in order to optically record from neurons in other ganglia that are affected by spike trains driven in the impaled neuron.

\subsubsection{Conventional Compound Microscope}

Once vibration-related noise is sufficiently minimized, the signalto-noise ratio is proportional to the square root of the light level (Zochowski et al. 2000b). Therefore, imaging is traditionally done using conventional compound microscopes (Fig. 5.4A), whose high NA objectives are much more light-efficient than those of stereomicroscopes. We use an Olympus BX51WI microscope, which was designed specifically for electrophysiology. Our lens of 
A Conventional microscope



FIGURE 5.4. Two microscope designs used for imaging. (A) For experiments involving minimal-to-no conventional intracellular recording we use a standard compound microscope. A parfocal digital camera is used to focus the PDA, and to provide a picture of the preparation to superimpose with the array data. (B) For experiments involving significant intracellular recording with the imaging we use a custom microscope consisting of the lower half of a compound microscope, to provide the bright transillumination needed for absorbance fVSDs, mated to and optically aligned with a stereomicroscope. A linear sliding lens switcher is used to alternate between a 0.6 NA $20 \times$ objective for the imaging, and a parfocal stereomicroscope objective for penetrating neurons with sharp electrodes. During experiments the imaging lens is fitted with a custom water immersion cap to eliminate image movement related to surface ripples. Both microscopes are mounted on motorized translators to allow the viewing/imaging area to be changed without disturbing impaled neurons. During experiments, the upper superstructure is further stabilized by inserting two foam-topped posts between the Gibraltar platform and the front corners of the lens switcher. A digital eyepiece camera is used with this microscope to obtain the images of the preparation to be superimposed with the array data.

choice is the Olympus XLUMPLFL20×W 0.95 NA water immersion objective. In Tritonia ganglia transilluminated with a $100 \mathrm{~W}, 12 \mathrm{~V}$ tungsten-halogen lamphouse and 0.9 NA condenser, this lens provides saturating light levels to the PDA with the lamp power set to slightly above half-maximum. High efficiency optics are important because they permit lower illumination intensities, translating to slower dye bleaching, lower phototoxicity concerns, and longer imaging time. With this microscope and optics we can obtain several $60 \mathrm{~s}$ acquisitions at near-saturating light levels for the PDA before dye bleaching begins to degrade the action potential signals.

\subsubsection{Custom Hybrid Microscope}

Despite their advantages, conventional compound microscopes lack stereopsis, making it difficult to incorporate multiple intracellular electrodes into imaging experiments. This is a major hindrance for many potential uses of optical recording, such as circuit mapping, where one would like to locate and immediately penetrate specific neurons identified in the optical recordings. Another aim is to drive a known neuron with one intracellular electrode while imaging its follower neurons, and then penetrate those with a second intracellular electrode. To carry out such experiments, we constructed a hybrid microscope that combines desirable features of both stereo and conventional compound microscopes (Fig. 5.4B).
We began by cutting off the top of a Nikon Optiphot microscope and then mounted a Zeiss SV-11 stereomicroscope onto a vertical post bolted to its stump. This allows the preparation to be viewed through the stereo objective, with the full depth perception necessary to penetrate specific neurons with intracellular electrodes. At the same time, we retained the compound microscope's lamphouse and condenser, to deliver maximum light to the preparation for imaging. A key part of this design is the linear sliding lens switcher (Combizoom 400, Kramer Scientific), which allows the user to alternate between a high NA objective for imaging and a conventional parfocal stereomicroscope objective for impaling neurons. This hybrid microscope design has been described in detail in a separate publication (Frost et al. 2007).

While the ability to integrate multiple intracellular electrodes into optical recording experiments is a big plus, the hybrid microscope has certain disadvantages compared with the traditional compound microscope. First, light transmission is lower through the zoom optics of the stereomicroscope body, requiring delivery of brighter illumination to the preparation to achieve adequate signal-to-noise ratios. To get sufficient illumination, we power a traditional $12 \mathrm{~V} 100 \mathrm{~W}$ tungsten halogen bulb with a $16 \mathrm{~V}$ racing car battery stepped down to $14.5 \mathrm{~V}$. This has the advantage of true DC power, and hence no ripple in the light to the imager. However, the brighter light comes at the cost of faster photobleaching and thus less imaging time per experiment (four to five $35 \mathrm{~s}$ acquisitions vs. several $60 \mathrm{~s}$ acquisitions with the conventional compound microscope setup). Second, the rear post mount of the stereomicroscope and PDA leads to increased vibration-based noise in the optical recordings. We minimize the vibration by inserting support posts beneath the front of the lens switcher, effectively supporting the microscope in three places. This design has greatly facilitated our use of multiple intracellular electrodes during imaging experiments. With intracellular recording comes the need to expose preparations to light while positioning electrodes and penetrating neurons. To avoid bleaching the fVSD while working with intracellular electrodes, we illuminate the preparation through a green filter that blocks the red wavelengths used for imaging with RH155.

\subsection{CORRELATING OPTICAL SIGNALS WITH SPECIFIC NEURONS IN THE PREPARATION}

Because of its poor spatial resolution, a PDA doesn't provide a useful image - its frame view mode looks like a hexagonal checkerboard of grayscale squares, corresponding to the brightness recorded by the different diodes at one point in time. Therefore, identifying the neurons the data are coming from is more difficult with a PDA than with camera-based imagers, which have higher spatial resolution. To do this, we take a picture of the preparation and superimpose it in Neuroplex with a diode map linked to the optical data. Clicking on any diode/neuron then displays the optical data recorded at that location (Fig. 5.3). The image superimposition involves a three-step procedure.

\subsubsection{Set the Camera and PDA Parfocal with One Another}

During experiments with the PDA, the focus point for the imaging is set using the live image provided by the microscope camera used to take the superimposed photograph. To insure that the PDA and camera are parfocal, we focus the camera on a calibration slide containing a black circle, and then adjust the focusing collar under the PDA so that the circle's edge shows the most abrupt 
black-to-white transition on the checkerboard pattern provided by the PDA. An alternative focusing method is to remove the PDA from its photoport and place a ground glass surface at the exact position the array face normally sits. Adjustments are then made to bring this image and that seen by the camera into parfocality. A third method is suggested by (Obaid et al. 2004), who used a custom microscope photoport component which, when slid into place, provides a direct view of the preparation image focused onto the photodiode array face.

\subsubsection{Obtain a Single, In-Focus Picture to Superimpose on the Array Data}

For three-dimensional neuronal preparations such as invertebrate ganglia, a single image taken through the objective lens will typically have just a portion of the surface neurons in focus. To obtain a clear picture of the entire visible ganglion surface to superimpose with the PDA data we take a stack of several images at different focal depths and then combine them into a single in-focus image (Fig. 5.5) using the fast focus enhancement feature of Rincon (ImagingPlanet, Goleta, CA).

\subsubsection{Accurately Align the Camera Picture with the PDA Data}

To correlate the imaging data with specific neurons, the above photograph must be precisely superimposed with the diode map. To do this, we collect both a camera picture and PDA readings of the light shining through three closely spaced pinholes in a foil sheet. The magnification and position of the camera image is then adjusted in Neuroplex until it exactly overlies the PDA image of the pinholes, rotating the PDA as needed, after which it is locked into position. In subsequent experiments these same coordinates can then be used to accurately superimpose the camera image of the preparation on the array data.

\subsection{GETTING GOOD SIGNALS}

When recording with fVSDs, neuronal action potentials may produce signals in the range of 0.001 to less than 0.0001 of the resting light level. Discriminating such small signals is a challenge. Here we describe some key steps that make this possible using the absorbance dye RH155 in the marine mollusks Tritonia diomedea and Aplysia californica.

\subsubsection{Choose an Optimal Transmission Filter}

The ability to see the small signals provided by voltage sensitive absorption dyes requires the use of bandpass filters optimized for each dye. RH155 and RH482, for example, have biphasic absorption curves - below a certain wavelength they decrease their absorption with depolarization, above it they increase absorption (Parsons et al. 1991; Jin et al. 2002). To avoid signal cancellation, the bandpass filter selected for use with such dyes must thus be centered on one side or the other of the crossover wavelength, with an appropriately limited bandwith. After trying several, we chose a 725/25 bandpass filter (Chroma Technology) for our work with Tritonia and Aplysia using RH155. Because the spectral characteristics of voltage sensitive dyes can shift in different solutions and tissues, the filter properties needed to provide optimal neuronal signals should be determined in the preparation of interest (Wang et al. 2009).

\subsubsection{Get the Light as Close to Saturation as Possible}

Once vibration-related noise has been sufficiently minimized (see below), signal-to-noise ratio increases in proportion to the square root of the illumination intensity (Zochowski et al. 2000b). This means that optimal optical recordings will be obtained when the resting light level reaching the imager is as close to the device's saturation level as possible. There are two steps for achieving this:
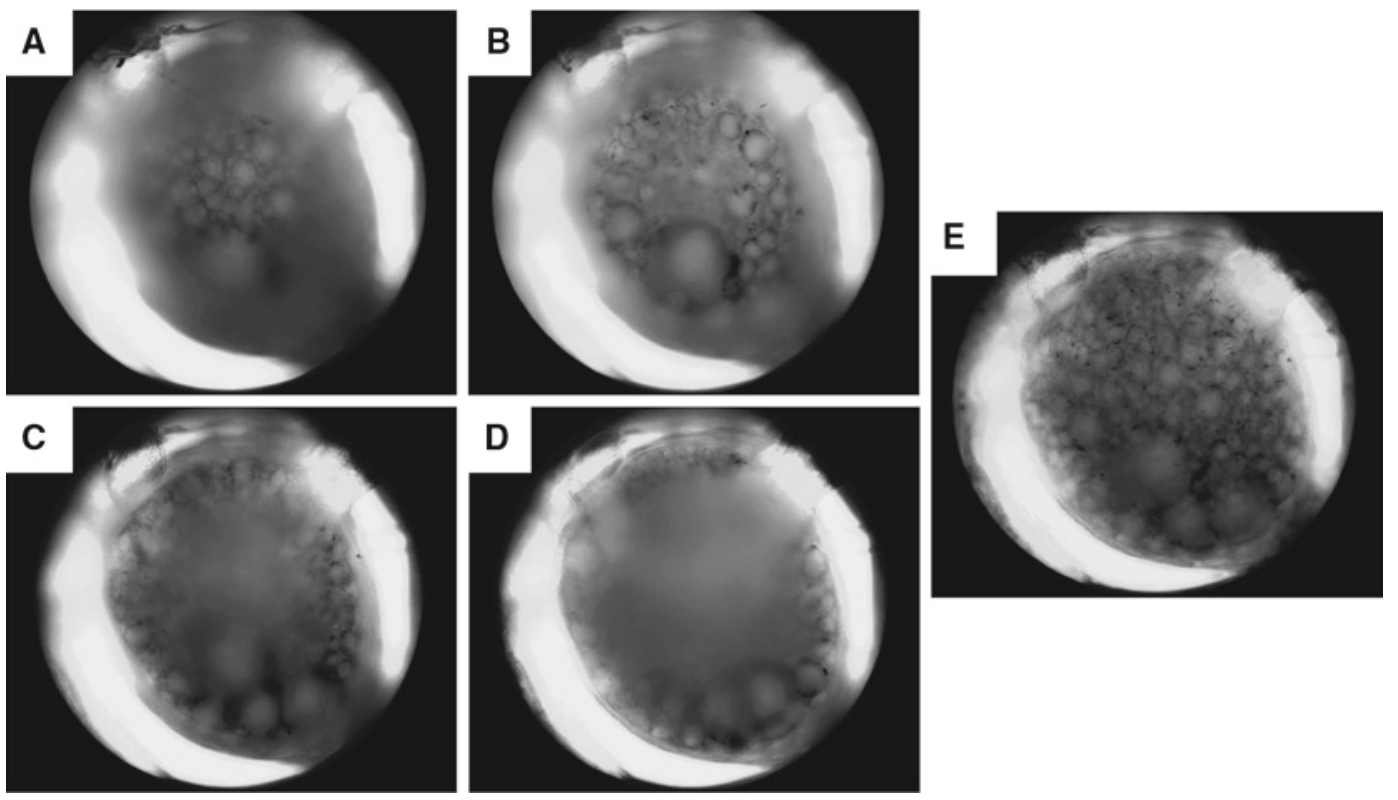



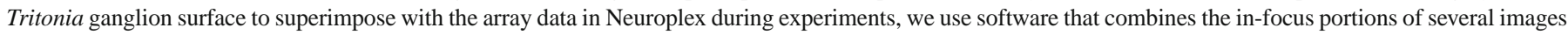


lower. (C) $200 \mu \mathrm{m}$ lower. (D) $300 \mu \mathrm{m}$ lower. (E) Combination of 13 images taken at $25 \mu \mathrm{m}$ intervals, showing all neurons visible on the ganglion surface. 
(1) maximize the light to the preparation, and (2) maximize the light transmitted from the preparation to the imager.

\subsubsection{Maximize the Light to the Preparation}

The lamphouse should be adjusted to position the bulb filament directly in the center of the field of view. If it has an adjustable back mirror, this should be positioned to make the image as bright as possible. Built-in diffusion and heat filters, the latter which attenuates the wavelength we use with RH155 (725 nm), can be removed from the light path between the lamphouse and the condenser to maximize light transmission. Alternatively, the built-in heat filter could be replaced with one chosen to block IR wavelengths while passing those needed for the particular dye being used. Different condensers concentrate the light into smaller or larger areas, reflecting the magnification range of the objectives they were designed to work with. The typical condenser illuminates an area of the recording chamber far larger than the area seen by the $20 \times$ objective we use in most experiments. After trying several condensers, the brightest light delivery so far was obtained with a simple $0.9 \mathrm{NA}$ Abbe achromat condenser having a swing-in top lens. This style is designed for use with low magnification objectives with the top lens removed, and higher magnification lenses with the top lens in place, which concentrates the light into a smaller area, closer to the field of view of our $20 \times$ imaging objective. The condenser height is empirically adjusted to produce the brightest illumination of the preparation, which occurs near the point of Kohler illumination, when the base diaphragm of the microscope is in sharp focus. With our preferred condenser, this occurs close enough to the preparation to require a recording chamber having a cover slip bottom topped by a layer of Sylgard just thick enough to hold the minute pins used to position the preparation. Under Kohler illumination, the microscope base diaphragm can be adjusted to constrict the illuminated area to the exact area being imaged. This prevents bleaching of other areas in the preparation, such as nearby ganglia, allowing them to be imaged later in the experiment.

\subsubsection{Maximize the Light from the Preparation to the Imager}

Increased efficiency in this part of the light path allows the desired near-saturating light levels to be obtained with lower lamp brightness, and thus slower dye bleaching and reduced potential phototoxicity. It also translates to longer imaging time, beneficial for monitoring long duration events such as the Tritonia swim motor program. An important way to maximize light transmission in this part of the pathway is to use the highest NA aperture objective lens available for the desired magnification, matched with a condenser with a comparable NA. A photoport that allows $100 \%$ of the light to be directed to the imager should also be used.

\subsubsection{Reduce Vibration-Related Noise}

The major source of noise in PDA experiments arises from vibration, which causes small oscillatory movements of contrast edges in the preparation on the photodiodes, producing oscillations in the recordings that can be many times the size of the optically recorded action potentials. Common sources of vibration include the opening of the shutter controlling the light to the preparation, pulsations related to perfusion pumps, and movements of the floor and walls caused by the building air handling systems, as well as by the movements of people in and outside the laboratory. A vibration isolation table is essential for minimizing externally-originating vibrations.
In addition, we (1) work on the ground floor, (2) use a compound microscope designed with low vibration and electrophysiology in mind (Olympus BX51WI), (3) use commercial water immersion objectives, or affix custom-made water immersion caps to our non-water immersion objectives to eliminate vibrations at the air/ solution interface, (4) mount the imaging shutter on the frame of the air table rather than on its surface, (5) ensure that cables connected to outside devices contact the table before reaching the microscope and imager, and (6) for the hybrid microscope, which otherwise would be supported only by the back mounting post, we insert two metal posts topped with foam pads between the frame of the lens switcher and the Gibraltar stage. The Neuroplex software can readily reveal the effects of these vibration-reducing efforts by displaying a Fourier analysis of the frequency components in the optical recordings.

\subsubsection{Issues Related to Preparations Requiring Cold Saline}

For preparations requiring cold saline, such as Tritonia $\left(11^{\circ} \mathrm{C}\right)$, condensation can form on the bottom side of the recording chamber, interfering with imaging. To prevent this, we place a drop of fluid on the condenser lens, which then fuses to the bottom of the recording chamber when the condenser is raised to its working position. In times of high humidity, significant condensation can also form on the inside of water immersion objective lenses, or on the inside of custom-made water immersion lens caps. During such conditions, we run a dehumidifier in the laboratory, and also place the objective and any lens cap in a dessicant-containing sealed plastic bag the night before the experiment. Just before recording, we attach the cap within the bag before placing the lens on the microscope. Our custom lens cap has a lightly greased O-ring within it to both hold it securely on the lens and to establish an airtight seal.

\subsubsection{Other Methods for Increasing the Number of Neurons Detected in the Imaging}

Although high-NA objective lenses are highly desirable for their light efficiency, this comes at the cost of a narrower depth of focus. This can be advantageous - the focus point of the microscope provides information on the depth location of the optically recorded neurons. A disadvantage is that signal loss from out-of-focus neurons means that many are not detected. A simple method to increase the number of neurons detected optically is to gently flatten the ganglion using a cover slip fragment held in place by Vaseline placed beside the ganglion on the chamber floor. In our experience, this simple procedure can double the number of neurons recorded optically.

\subsection{SPIKE-SORTING THE RAW OPTICAL DATA WITH INDEPENDENT COMPONENT ANALYSIS}

Using the above techniques, we routinely obtain simultaneous optical recordings of several dozen to well over 100 neurons from the dorsal surface of the Tritonia pedal ganglion. Many scientific issues can be addressed by simply inspecting the filtered, but otherwise, raw diode recordings. For circuit mapping, previously unknown neurons that fire during a given motor program are easily identified. Neurons excited or inhibited by neurons driven with intracellular electrodes are also easily spotted. Other issues, however, cannot readily be addressed through simple visual inspection of the data traces. For example, it is nearly impossible to determine 
A

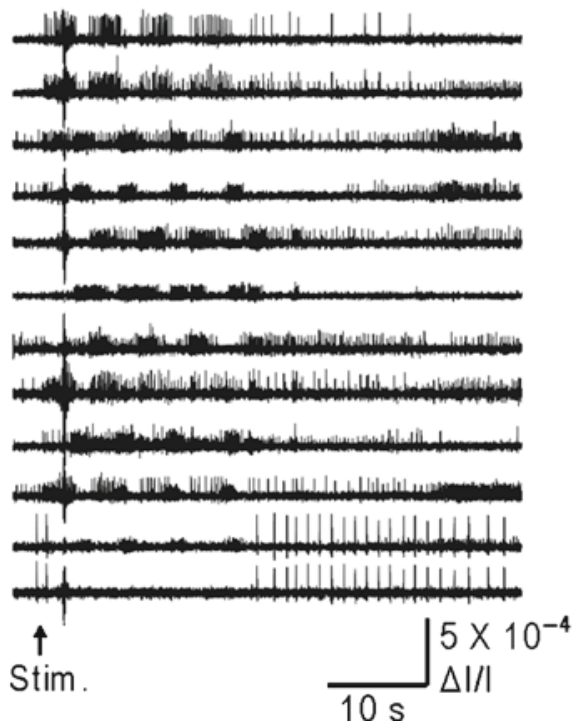

B1 Independent components



B2





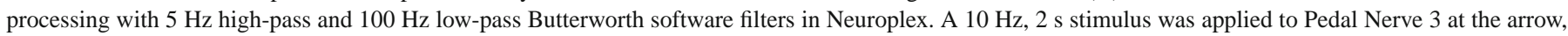

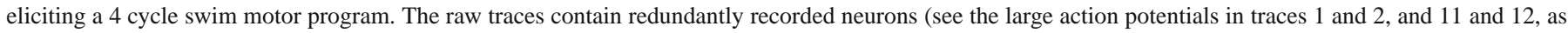

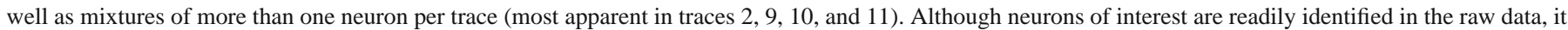





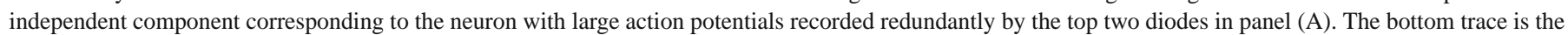

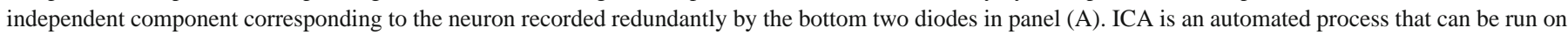



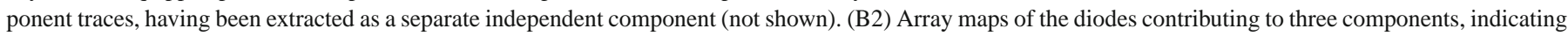
the XY position and approximate size of the source neurons.

the number of neurons present in a typical optical recording. The larger neurons appear redundantly in many traces, while many traces contain action potentials from multiple neurons (Fig. 5.6A). To take full advantage of the power of optical recording, we need a method for spike sorting these mixed and redundant data sets.

For several years beginning in the late 1980s, Larry Cohen and colleagues applied a spike template matching method for sorting the redundant and mixed raw spike data into a new set of traces consisting of one unique neuron per trace (Zecevic et al. 1989; Falk et al. 1993; Tsau et al. 1994; Wu et al. 1994a, Wu et al. 1994b; Hickie et al. 1997; Zochowski et al. 2000a). A downside of this method was its heavy reliance on experimenter judgment, leading to a typical processing time of several days per record (Cohen et al. 1989). More recently, Independent Component Analysis (ICA) has been introduced as a rapid and automated method for spike-sorting optical recording data sets obtained with fVSDs (Brown et al. 2001; Brown et al. 2008). ICA is a blind source separation methodology for separating signal mixtures into their original sources. ICA finds a linear transformation of the raw traces that minimizes the mutual information shared between the output components, separating the redundant and mixed neuronal source signals into separate, single-neuron traces (Fig. 5.6B1). For an imager with $N$ pixels or diodes, ICA can separate up to $N$ independent components, which can include artifacts and other noise sources, effectively removing them from the neuronal recordings (Brown et al. 2001).

We used infomax ICA (Bell and Sejnowski 1995; Delorme and Makeig 2004). With a HP Xeon $3.2 \mathrm{GHz}$ Z400 workstation with 8 GB RAM running Windows XP Professional x64, ICA can process a $35 \mathrm{~s}$ file of 464 optical traces digitized at $1,600 \mathrm{~Hz}$ into the set of independent components in as little as 9 min. MATLAB programs have also been written (C.M.-K.) that return maps showing the position of each resolved component on the diode array (Fig. 5.6B2).

Using ICA we are able to (1) eliminate redundancy by combining neurons recorded by multiple diodes into single traces, (2) sort multiple neurons recorded by single diodes into separate singleneuron traces, (3) provide the array location of each resolved neuron, and (4) remove artifacts from the traces. ICA thus represents a powerful, fast, and automated tool for spike sorting data sets obtained with photodiode arrays and fast voltage sensitive dyes.

\section{ACKNOWLEDGMENTS}

Supported by NS060921, Dart Foundation and Grass Foundation Marine Biological Laboratory summer fellowships, the Fred B. Snite Foundation, Rosalind Franklin University of Medicine and Science (WF), and the Howard Hughes Medical Institute (TS). We thank JY $\mathrm{Wu}, \mathrm{LB}$ Cohen and L Eliot for comments on the manuscript.

\section{REFERENCES}

Bell AJ, Sejnowski TJ (1995) An information-maximization approach to blind separation and blind deconvolution. Neural Comput 7:1129-1159.

Boyle MB, Cohen LB, Macagno ER, Orbach H (1983) The number and size of neurons in the CNS of gastropod molluscs and their suitability for optical recording of activity. Brain Res 266:305-317.

Brown GD, Yamada S, Sejnowski TJ (2001) Independent component analysis at the neural cocktail party. Trends Neurosci 24:54-63. 
Brown GD, Yamada S, Nakashima M, Moore-Kochlacs C, Sejnowski TJ (2008) Independent component analysis of optical recordings from Tritonia swimming neurons. In: Technical Report INC-08-001, Institute for Neural Computation, University of California at San Diego.

Carlson GC, Coulter DA (2008) In vitro functional imaging in brain slices using fast voltage-sensitive dye imaging combined with whole-cell patch recording. Nat Protoc 3:249-255.

Chang PY, Jackson MB (2003) Interpretation and optimization of absorbance and fluorescence signals from voltage-sensitive dyes. J Membr Biol 196:105-116.

Cohen L, Hopp HP, Wu JY, Xiao C, London J (1989) Optical measurement of action potential activity in invertebrate ganglia. Annu Rev Physiol 51:527-541.

Delorme A, Makeig S (2004) EEGLAB: an open source toolbox for analysis of single-trial EEG dynamics including independent component analysis. J Neurosci Methods 134:9-21.

Ebner TJ, Chen G (1995) Use of voltage-sensitive dyes and optical recordings in the central nervous system. Prog Neurobiol 46:463-506.

Falk CX, Wu J, Cohen LB, Tang AC (1993) Nonuniform expression of habituation in the activity of distinct classes of neurons in the Aplysia abdominal ganglion. J Neurosci 13:4072-4081.

Frost WN, Wang J, Brandon CJ (2007) A stereo-compound hybrid microscope for combined intracellular and optical recording of invertebrate neural network activity. J Neurosci Methods 162:148-154.

Greenberg DS, Houweling AR, Kerr JN (2008) Population imaging of ongoing neuronal activity in the visual cortex of awake rats. Nat Neurosci 11:749-751.

Grinvald A, Salzberg BM, Cohen LB (1977) Simultaneous recording from several neurones in an invertebrate central nervous system. Nature (Lond) 268:140-142.

Hickie C, Cohen LB, Balaban PM (1997) The synapse between LE sensory neurons and gill motoneurons makes only a small contribution to the Aplysia gill-withdrawal reflex. Eur J Neurosci 9:627-636.

Jin W, Zhang RJ, Wu JY (2002) Voltage-sensitive dye imaging of population neuronal activity in cortical tissue. J Neurosci Methods 115:13-27.

Kerr JN, Greenberg D, Helmchen F (2005) Imaging input and output of neocortical networks in vivo. Proc Natl Acad Sci U S A 102:14063-14068.

Kojima S, Hosono T, Fujito Y, Ito E (2001) Optical detection of neuromodulatory effects of conditioned taste aversion in the pond snail Lymnaea stagnalis. J Neurobiol 49:118-128.

Kosmidis EK, Cohen LB, Falk CX, Wu JY, Baker BJ (2005) Imaging with voltage-sensitive dyes: spike signals, population signals, and retrograde transport. In: Yuste R, Konnerth A (eds) Imaging in neuroscience and development. A laboratory manual. Cold Spring Harbor Laboratory Press, Cold Spring Harbor, NY.

London JA, Zecevic D, Cohen LB (1987) Simultaneous optical recording of activity from many neurons during feeding in Navanax. J Neurosci 7:649-661.

Momose-Sato Y, Sato K et al. (1999) Evaluation of voltage-sensitive dyes for long-term recording of neural activity in the hippocampus. J Membr Biol 172:145-157.

Nakashima M, Yamada S, Shiono S, Maeda M, Satoh F (1992) 448-Detector optical recording system: development and application to Aplysia gillwithdrawal reflex. IEEE Trans Biomed Eng 39:26-36.

Neunlist M, Peters S, Schemann M (1999) Multisite optical recording of excitability in the enteric nervous system. Neurogastroenterol Motil 11:393-402.

Nikitin ES, Balaban PM (2000) Optical recording of odor-evoked responses in the olfactory brain of the naive and aversively trained terrestrial snails. Learn Membr 7:422-432.

Obaid AL, Koyano T, Lindstrom J, Sakai T, Salzberg BM (1999) Spatiotemporal patterns of activity in an intact mammalian network with single-cell resolution: optical studies of nicotinic activity in an enteric plexus. J Neurosci 19:3073-3093.

Obaid AL, Loew LM, Wuskell JP, Salzberg BM (2004) Novel naphthylstyrylpyridium potentiometric dyes offer advantages for neural network analysis. J Neurosci Methods 134:179-190.

Parsons TD, Salzberg BM, Obaid AL, Raccuia-Behling F, Kleinfeld D (1991) Long-term optical recording of patterns of electrical activity in ensembles of cultured Aplysia neurons. J Neurophysiol 66:316-333.

Salzberg BM, Grinvald A, Cohen LB, Davila HV, Ross WN (1977) Optical recording of neuronal activity in an invertebrate central nervous system: simultaneous monitoring of several neurons. J Neurophysiol 40:1281-1291.

Sato TR, Gray NW, Mainen ZF, Svoboda K (2007) The functional microarchitecture of the mouse barrel cortex. PLoS Biol 5:e189.

Schemann M, Michel K, Peters S, Bischoff SC, Neunlist M (2002) Cuttingedge technology. III. Imaging and the gastrointestinal tract: mapping the human enteric nervous system. Am J Physiol Gastrointest Liver Physiol 282:G919-G925.

Senseman DM (1996) High-speed optical imaging of afferent flow through rat olfactory bulb slices: voltage-sensitive dye signals reveal periglomerular cell activity. J Neurosci 16:313-324.

Sinha SR, Saggau P (1999) Optical recording from populations of neurons in brain slices. In: Johanson $\mathrm{H}$, Windhorst $\mathrm{U}$ (eds) Modern techniques in neuroscience research. Springer Verlag, Berlin.

Stosiek C, Garaschuk O, Holthoff K, Konnerth A (2003) In vivo two-photon calcium imaging of neuronal networks. Proc Natl Acad Sci U S A 100: 7319-7324.

Takahashi N, Sasaki T, Usami A, Matsuki N, Ikegaya Y (2007) Watching neuronal circuit dynamics through functional multineuron calcium imaging (fMCI). Neurosci Res 58:219-225.

Tsau Y, Wu J, Hopp H, Cohen LB, Schiminovich D, Falk CX. (1994) Distributed aspects of the response to siphon touch in Aplysia: spread of stimulus information and cross-correlation analysis. J Neurosci 14:4167-4184.

Vanden Berghe P, Bisschops R, Tack J (2001) Imaging of neuronal activity in the gut. Curr Opin Pharmacol 1:563-567.

Wang Y, Jing G, Perry S, Bartoli F, Tatic-Lucic S (2009) Spectral characterization of the voltage-sensitive dye di-4-ANEPPDHQ applied to probing live primary and immortalized neurons. Opt Express 17:984-990.

Wu J, Cohen LB, Falk CX (1994a) Neuronal activity during different behaviors in Aplysia: a distributed organization? Science 263:820-823.

Wu J, Tsau Y, Hopp H, Cohen LB, Tang AC, Falk CX (1994b) Consistency in nervous systems: trial-to-trial and animal-to-animal variations in the responses to repeated applications of a sensory stimulus in Aplysia. J Neurosci 14: $1366-1384$.

Yagodin S, Collin C, Alkon DL, Sheppard NF Jr, Sattelle DB (1999) Mapping membrane potential transients in crayfish (Procambarus clarkii) optic lobe neuropils with voltage-sensitive dyes. J Neurophysiol 81:334-344.

Yang S, Doi T, Asako M, Matsumoto-Ono A, Kaneko T, Yamashita T (2000) Multiple-site optical recording of mouse brainstem evoked by vestibulocochlear nerve stimulation. Brain Res 877:95-100.

Yuste R (2008) Circuit neuroscience: the road ahead. Front Neurosci 2:6-9.

Zecevic D, Wu J, Cohen LB, London JA, Hopp H, Falk CX (1989) Hundreds of neurons in the Aplysia abdominal ganglion are active during the gill-withdrawal reflex. J Neurosci 9:3681-3689.

Zochowski M, Cohen LB, Fuhrmann G, Kleinfeld D (2000a) Distributed and partially separate pools of neurons are correlated with two different components of the gill-withdrawal reflex in Aplysia. J Neurosci 20:8485-8492.

Zochowski M, Wachowiak M, Falk CX, Cohen LB, Lam YW, Antic S, Zecevic D (2000b) Imaging membrane potential with voltage-sensitive dyes. Biol Bull 198:1-21. 